PROF. SHIDA YOUSEFI (Orcid ID : 0000-0002-9855-4305)

DR. DAGMAR SIMON (Orcid ID : 0000-0001-8965-9407)

Article type : Letter to the Editor

\title{
Dupilumab reduces inflammation and restores the skin barrier in patients with atopic dermatitis
}

Maria Helena Rohner ${ }^{1, *}$, Kathrin Thormann ${ }^{1, *}$, Simone Cazzaniga ${ }^{1,2}$, Shida Yousefi ${ }^{3}$, HansUwe Simon $^{3,4}$, Christoph Schlapbach ${ }^{1}$, Dagmar Simon ${ }^{1}$

1Department of Dermatology, Inselspital, Bern University Hospital, University of Bern, Bern, Switzerland

${ }^{2}$ Centro Studi GISED, Bergamo, Italy

IInstitute of Pharmacology, University of Bern, Bern, Switzerland

${ }^{4}$ Department of Clinical Immunology and Allergology, Sechenov University, Moscow, Russia

*These authors equally contributed to the study.

Correspondence:

Dagmar Simon, MD

Department of Dermatology, Inselspital, CH-3010 Bern, Switzerland 
Tel.: +41 31632 2278; E-mail: dagmar.simon@insel.ch

ORCID: https:// orcid.org/0000-0001-8965-9407

\section{AUTHOR CONTRIBUTION}

SY, HUS, CS and DS designed the study, MHR, KT and SC did the data collection and analyses, and drafted the manuscript, SY, HUS, CS and DS supervised the project and revised the manuscript.

\section{KEY WORDS}

atopic dermatitis, dupilumab, epidermal barrier, interleukin-13, type 2 inflammation

Words: 600

Figures: 2; Supporting information: 5 pages

This article is protected by copyright. All rights reserved 
To the Editor,

The pathogenesis of atopic dermatitis (AD), a common chronic inflammatory skin disease, is based on a genetic predisposition that determines both skin barrier function and type 2 immune reaction ${ }^{1,2, ~ S 1-4}$. Type 2 cytokines may further impair the epidermal barrier and trigger AD inflammation. ${ }^{1}$

Therapy with dupilumab that inhibits the action of interleukin (IL)-4 and IL-13 by blocking the shared receptor, results in a rapid improvement of symptoms, clinical signs and quality of life in AD patients. ${ }^{3,}$ s5-11 Transcriptome analyses of skin samples suggest that it affects mRNA expression of genes related to type 2 inflammation, T helper (Th)17/Th22 pathways, dendritic cells, epidermal barrier and lipid metabolism. 4, 5, S12-13

This study aimed at assessing the efficacy and safety of dupilumab therapy in patients with $A D$ referred to a tertiary center and at investigating the effect of dupilumab on both inflammation and epidermal barrier by applying immunofluorescence techniques. It has been approved by the Ethics committee of the Canton Bern.

In this retrospective study, data of 34 patients with AD under dupilumab therapy (10 females, mean age $40.1 \pm 12.4$ years) were included (Table S1). All patients had severe AD with a mean SCORAD of $64.2 \pm 18.5$ at baseline. Patients were treated with a loading dose of 600 mg dupilumab followed by $300 \mathrm{mg}$ every 2 weeks.

In our patient cohort, dupilumab therapy resulted in a significant improvement of clinical signs and symptoms (Table S2). The percentage of patients achieving physician global assessment (PGA) 0-1 was $47 \%$ after 4 months and remained unchanged over time. Remarkably, our results are comparable with those of clinical and real-life studies, although exclusively patients with severe AD were eligible to get dupilumab therapy. ${ }^{55-11, ~ \$ 14, ~} \$ 15$ Already one month after initiating dupilumab therapy, $60 \%$ reported that $A D$ did not or minimally affected their quality of life (Figure 1).

This article is protected by copyright. All rights reserved 
All ten patients with initial overlapping immunosuppressives plus dupilumab therapy were able to stop immunosuppressives during the first 3-4 months (Table S3). When PGA 0-1 was achieved and maintained for 2 to 3 months, we attempted to increase the intervals between injections. As a result, 12 patients were able to apply dupilumab every 3 to 4 weeks without loss of efficacy.

Overall, dupilumab therapy was well tolerated. The frequency of dupilumab-associated conjunctivitis was $32 \%$. One AD patient with concomitant keratoconus discontinued therapy after developing severe keratoconjunctivitis. A longitudinal analysis revealed that worsening of SCORAD or DLQI was associated with $A D$ onset in adulthood, presence of specific comorbidities, severe AD, and sleep loss (Table S4).

From eight patients responding to dupilumab without concomitant immunosuppressive therapy, specimens of AD skin taken before and 6-8 weeks after initiating dupilumab therapy were available. Upon dupilumab therapy, a significant decrease in numbers of inflammatory cells, and, concomitantly, reduced numbers of cytokines involved in the pathogenesis of AD (IL-5, IL-9, IL-13, IL-15, IL-17, IL-22, IFN- $\gamma$ ) were observed (Figure 2). We noted a reduction toward or even below levels found in non-lesional skin. Type 2 cytokines are known to block the production of proteins required for skin barrier formation. 6 , s16-17 In parallel with the decreased inflammation, a significant increased expression of filaggrin, the protease inhibitor Lympho-epithelial Kazal-type-related inhibitor (LEKTI) and the antimicrobial peptides human beta-defensin (HBD)-3 and cathelicidin LL-37 was found. The improved skin barrier is assumed to result in a decreased expression of epidermal cytokines. Indeed, the epidermal expression of the tissue alarmins (TSLP, IL-15 and IL-25) significantly declined. By inhibiting TSLP, IL-15, IL-25 and IL-33 release, type 2 inflammation is likely further downregulated.

Collectively, we provide further evidence that dupilumab breaks the vicious cycle of IL-13driven inflammation and barrier dysfunction in the skin resulting in a significant improvement of $A D$ and increase in patients' quality of life. 


\section{ACKNOWLEDGEMENTS}

We thank Eleonora Valente, Evelyne Kozlowski and Riim Nasser for technical assistance. Images were acquired on equipment supported by the Microscopy Imaging Centre of the University of Bern.

\section{FUNDING INFORMATION}

Research of HUS and SY is supported by grants of the Swiss National Science Foundation (grant numbers 310030_184816 to H.U.S. and 31003A_173215 to S.Y.). CS is supported by the Peter Hans Hofschneider Professorship.

\section{CONFLICTS OF INTEREST}

MHR, SC, SY, and HUS have no conflicts of interest to declare. CS has received honoraria as adviser for AbbVie, LEO Pharma, Lilly, and Novartis and has received research funding from PPM Services. DS has been an investigator, advisory board member, or consultant for AbbVie, AstraZeneca, Galderma, Lilly, Pfizer, Roche Pharma, Sanofi Genzyme.

\section{Maria Helena Rohner ${ }^{1, *}$}

Kathrin Thormann ${ }^{1, *}$ 
Simone Cazzaniga ${ }^{1,2}$

Shida Yousefi ${ }^{3}$

Hans-Uwe Simon ${ }^{3,4}$

Christoph Schlapbach ${ }^{1}$

Dagmar Simon ${ }^{1}$

${ }^{1}$ Department of Dermatology, Inselspital, Bern University Hospital, University of Bern, Bern, Switzerland

${ }^{2}$ Centro Studi GISED, Bergamo, Italy

3Institute of Pharmacology, University of Bern, Bern, Switzerland

${ }^{4}$ Department of Clinical Immunology and Allergology, Sechenov University, Moscow, Russia

${ }^{*}$ These authors equally contributed to the study.

This article is protected by copyright. All rights reserved 


\section{REFERENCES}

1. Simon D, Wollenberg A, Renz H, Simon HU. Atopic Dermatitis: Collegium Internationale Allergologicum (CIA) Update 2019. Int Arch Allergy Immunol 2019;178:207-218.

2. Akdis CA, Akdis M, Simon D et al. T cells and T cell-derived cytokines as pathogenic factors in the nonallergic form of atopic dermatitis. J Invest Dermatol 1999;113:628-634.

3. Simpson EL, Bieber T, Guttman-Yassky E et al. Two Phase 3 Trials of Dupilumab versus Placebo in Atopic Dermatitis. N Engl J Med 2016;375:2335-2348.

4. Guttman-Yassky E, Bissonnette R, Ungar B et al. Dupilumab progressively improves systemic and cutaneous abnormalities in patients with atopic dermatitis. J Allergy Clin Immunol 2019;143:155-172.

5. Hamilton JD, Suárez-Fariñas $\mathrm{M}$, Dhingra $\mathrm{N}$ et al. Dupilumab improves the molecular signature in skin of patients with moderate-to-severe atopic dermatitis. $\mathrm{J}$ Allergy Clin Immunol 2014;134:1293-1300.

6. Howell MD, Kim BE, Gao P et al. Cytokine modulation of atopic dermatitis filaggrin skin expression. J Allergy Clin Immunol 2007;120:150-155.

\section{SUPPORTING INFORMATION}

Additional supporting information may be found online in the Supporting Information section. 


\section{FIGURE LEGENDS}

FIGURE 1 Dupilumab therapy improves disease severity and quality of life. Graphs show mean and individual values of the total SCORAD, and percentage of patients in defined ranges of physician global assessment (PGA) score and dermatology life quality index (DLQI) at baseline and follow-up over 24 months. PGA is scored 0-1, clear, almost clear; 2-

3, mild to moderate; 4, severe atopic dermatitis. DLQI categories are $<6$, no and mild; 6-10, moderate; $11+$, large impact on quality of life.

FIGURE 2 Dupilumab reduces inflammation and restores the epidermal barrier. Graphs and representative images show the numbers of inflammatory cells ( $T$ cells, eosinophils, mast cells and CD1a+ epidermal dendritic cells) and cytokines (IL-5, IL-9, IL-13, IL-15, IL-17, IL-22, IFN- $\gamma$ ), epithelial barrier proteins (filaggrin, lymphoepithelial-Kazal-type-related inhibitor (LEKTI), human $\beta$-defensin (HBD)-3 and cathelicidin LL-37), as well as cytokines expressed by keratinocytes (TSLP, IL-15, IL-25, IL-33) in lesional atopic dermatitis skin before (A) and after (B) dupilumab therapy as well as non-lesional (C) and normal (D) skin. Magnifications x400 (CD1a, epidermal barrier proteins), x630 (other cell types, cytokines). *, $p<0.05 ;{ }^{* *}, p<0.01 ;{ }^{* * *}, p<0.001 ;{ }^{* * *}, p<0.0001$.

This article is protected by copyright. All rights reserved 

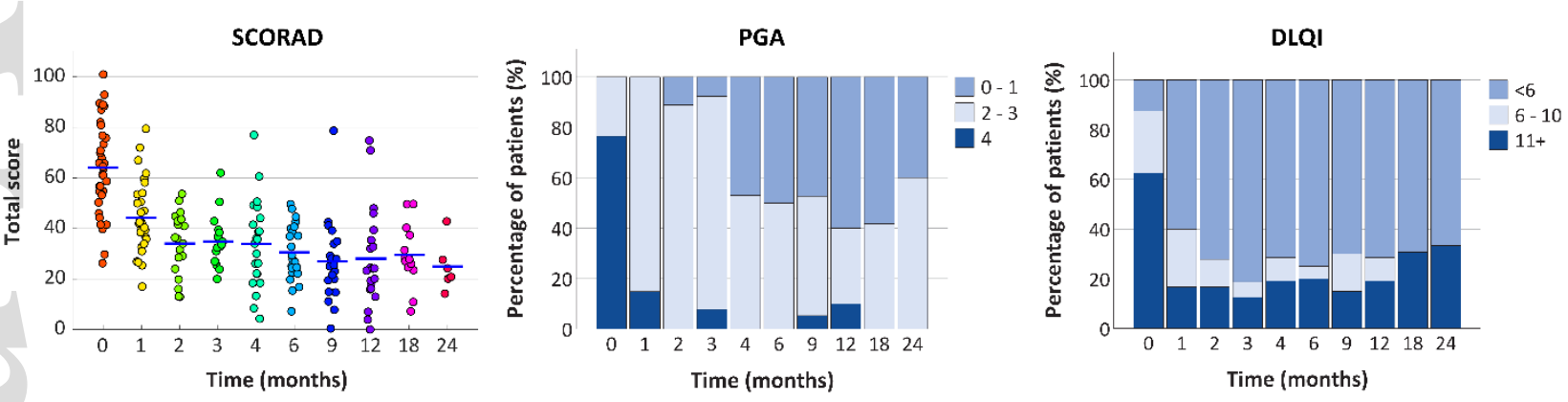

Figure 1

This article is protected by copyright. All rights reserved 

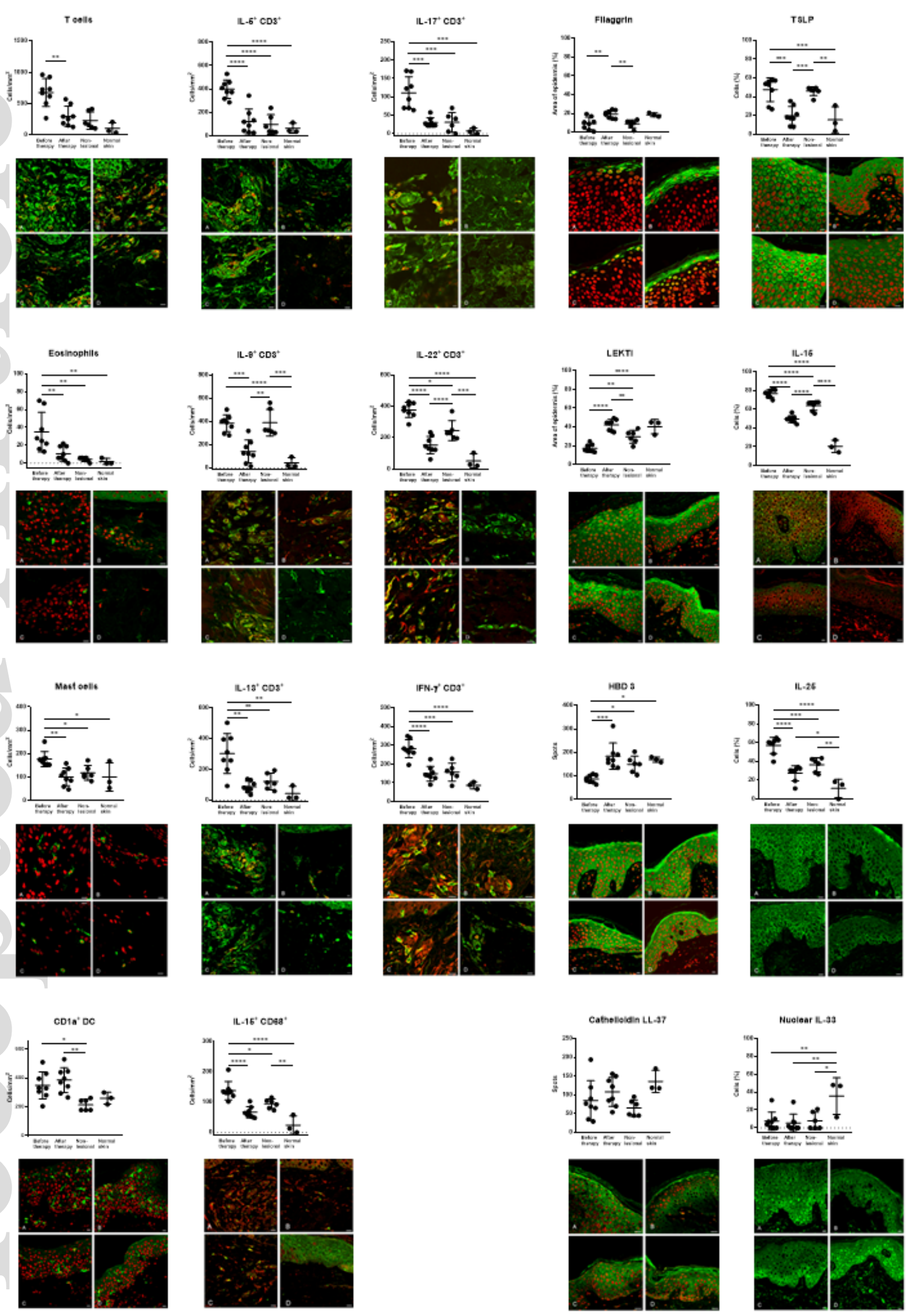

Figure 2

This article is protected by copyright. All rights reserved 